\title{
Ganzenmüller, Jörg (Hrsg.): Europas vergessene Diktaturen. Diktatur und Diktaturüberwindung in Spanien, Portugal und Griechenland, 288 S., Böhlau, Köln u. a. 2018.
}

\author{
Jan C. Behrends \\ Angenommen: 17. Dezember 2020 / Online publiziert: 5. Januar 2021 \\ (C) Der/die Autor(en) 2021
}

Die historische Forschung zu modernen Diktaturen in Europa beschäftigt sich in der Regel primär mit dem Kommunismus, dem italienischen Faschismus oder dem Nationalsozialismus und ihren jeweiligen Folgen. Der vorliegende Band, der dem Andenken an Hans-Peter Schwarz gewidmet ist, hat das Anliegen, die Diktaturerfahrungen in Spanien, Portugal und Griechenland zu analysieren. Diese südeuropäischen Diktaturen stehen besonders aus deutscher Perspektive im Schatten der eigenen Geschichte, die durch Nationalsozialismus und Kommunismus geprägt war. Gerade in einer Zeit, in der die liberale Demokratie in Europa von vielen Seiten unter Druck gerät, populistische Bewegungen Zulauf bekommen und autoritäre Herrscher bewundert werden, ist es wichtig, die Vielfalt der Diktaturerfahrungen zu betonen und Wege in die Demokratie zu würdigen. Denn die Geschichte der Nachkriegszeit in Europa war keineswegs nur eine Geschichte der Demokratie. Diktatur und autokratische Herrschaft existierten vor 1989 nicht nur in Osteuropa.

Einleitend skizziert Adam Krzemiński am Beispiel Osteuropas, warum die liberale Ordnung auf dem Kontinent gegenwärtig bedroht ist. Anschließend gliedert sich der Band in drei Teile: zuerst werden die Grundzüge der südeuropäischen Diktaturen nach 1945 in drei Beiträgen vorgestellt. Dabei stehen Fragen nach der jeweiligen Ideologie, der Herrschaftspraxis und der Gewalt im Vordergrund. Carlos Collado Seidel eröffnet die Aufsätze zu südeuropäischen Diktaturen mit seinem Beitrag über Spanien unter General Francisco Franco. Er betont die Spaltung der spanischen Gesellschaft durch Bürgerkrieg und exzessive Gewalt, verweist auf das Bündnis zwischen katholischer Kirche und Franco und erklärt die Funktionsweise des franquistischen Führerstaates. Collado Seidel erläutert auch, dass die Öffnung des Landes bereits zur Zeit der Diktatur begann. Die langlebigste rechte Diktatur in Europa war das Salazar-Regime in Portugal. Außenpolitisch war das autoritäre Portugal enger mit den Westmächten verbunden als das franquistische Spanien. Christiane Abele unterstreicht in ihrem Beitrag, dass der wirtschaftliche Korporatismus eine wichtige Grundlage des Regimes in Portugal war. Nationalismus und Antikom-

J. C. Behrends ( $(\nabla)$

Leibniz-Zentrum für Zeithistorische Forschung Potsdam, Potsdam, Deutschland

E-Mail: behrends@zzf-potsdam.de 
munismus bildeten die ideologischen Stützen. Letztlich scheiterte der Salazarismus unter anderem an seiner Unfähigkeit, die Konflikte in den Kolonien zu beenden. In Griechenland dauerte die Militärdiktatur von 1967 bis 1974. Janis Nabaldidacis hebt in seinem Beitrag hervor, dass sich das Militärregime in Athen grundlegend von den Diktaturen in Portugal und Spanien unterschied. Es war von kurzer Dauer und es mangelte ihm an ideologischer Legitimation. Schlussendlich konnte es sich nur auf Gewalt stützen; eine Konsolidierung der Herrschaft gelang nicht.

Der zweite Teil des Bandes behandelt die Systemtransformation in Südeuropa. Die erfolgreiche Etablierung von Demokratien in diesen Staaten ist gegenwärtig auch deshalb von Interesse, weil die Bilanz der postkommunistischen Gesellschaften drei Jahrzehnte nach 1989 wesentlich gemischter ausfällt. Wie können wir diese Unterschiede erklären? Spanien gelang es nach Francos Tod, sich innerhalb kurzer Zeit in die Strukturen des Westens zu integrieren. Innenpolitisch wurde die jüngere Geschichte zu einem Tabuthema, das weitgehend verdrängt wurde. Nach der Erfahrung von Bürgerkrieg und Diktatur war die spanische Elite im Übergang zur Demokratie auf Ausgleich und Konsens bedacht. Die Konflikte der Vergangenheit sollten nicht weiter die Geschicke des Landes bestimmen. Die Verbrechen der Franco-Zeit blieben aufgrund einer Amnestie ungesühnt. In Portugal endete die Diktatur mit der „Nelkenrevolution“ - und damit mit einem friedlichen Umbruch wie Europa bis dahin nur wenige gekannt hatte. Beim Sturz des Regimes spielten die Streitkräfte eine entscheidende Rolle. Die Demokratisierung, so zeigt Antonia Muńoz Sánchez, mussten aber politische Kräfte leisten. In der Auseinandersetzung zwischen linker Massenbewegung und konservativen Kräften formierte sich die Gesellschaft neu. Beim Meistern der Krise halfen die Wirtschaftshilfen aus Brüssel und Bonn maßgeblich. Für Griechenland interpretiert Adamantios Theodor Skordos das Ende des Obristenregimes als eigentlichen Schlusspunkt des Bürgerkrieges. Der Verfasser betont die Verbindung zur Zypernfrage und die hohe Geschwindigkeit der Liberalisierung eines Regimes, das von Beginn an nur über wenig Rückhalt verfügte.

Der letzte Teil des Bandes beschäftigt sich mit der Aufarbeitung der südeuropäischen Diktaturen. In Spanien dominierte bis weit in die 1990er Jahre hinein das Beschweigen der franquistischen Verbrechen und auch in Portugal überwog - nach einer kurzen Phase der Abrechnung mit dem Estado Novo - schon bald die Zurückhaltung bei der historischen Aufarbeitung. Erst im 21. Jahrhundert nahm die Auseinandersetzung mit der Diktatur wieder Fahrt auf. Insgesamt handelt es sich bei der vorliegenden Publikation um einen anregenden und überzeugenden Band, der ein Thema und eine Region behandelt, die in Deutschland wenig Interesse erregen. $\mathrm{Zu}$ Unrecht, wie sich hier zeigt. In Zukunft wäre es interessant, die Transformationsprozesse nach dem Ende der Diktaturen in Süd- und Osteuropa zu vergleichen. So könnten wir die unterschiedlichen Wege, die Gesellschaften nach dem Ende von Unterdrückung und Gewalt gegangen sind, besser verstehen.

Funding Open Access funding enabled and organized by Projekt DEAL.

Open Access Dieser Artikel wird unter der Creative Commons Namensnennung 4.0 International Lizenz veröffentlicht, welche die Nutzung, Vervielfältigung, Bearbeitung, Verbreitung und Wiedergabe in jeglichem Medium und Format erlaubt, sofern Sie den/die ursprünglichen Autor(en) und die Quelle ord- 
nungsgemäß nennen, einen Link zur Creative Commons Lizenz beifügen und angeben, ob Änderungen vorgenommen wurden.

Die in diesem Artikel enthaltenen Bilder und sonstiges Drittmaterial unterliegen ebenfalls der genannten Creative Commons Lizenz, sofern sich aus der Abbildungslegende nichts anderes ergibt. Sofern das betreffende Material nicht unter der genannten Creative Commons Lizenz steht und die betreffende Handlung nicht nach gesetzlichen Vorschriften erlaubt ist, ist für die oben aufgeführten Weiterverwendungen des Materials die Einwilligung des jeweiligen Rechteinhabers einzuholen.

Weitere Details zur Lizenz entnehmen Sie bitte der Lizenzinformation auf http://creativecommons.org/ licenses/by/4.0/deed.de.

\section{Kümmel, Verena: Vergangenheit begraben? Die gestohlenen Leichen Mussolinis und Pétains und der Kampf um die Erinnerung, 370 S., Böhlau, Köln u.a. 2018.}

\section{Claudia C. Gatzka}

Angenommen: 17. Dezember 2020 / Online publiziert: 5. Januar 2021

(C) Der/die Autor(en) 2021

Wie bestatten Demokratien einen abgesetzten Diktator? Mit dieser Frage schließt Verena Kümmel die politisch gespaltenen Nachkriegsgesellschaften Frankreichs und Italiens auf. Es lag in der Verantwortung der neuen, aus Résistance und Resistenza hervorgegangenen Regierungen, die einstigen Regimeführer Benito Mussolini und Philippe Pétain zu Grabe zu tragen. Die Art der Bestattung, so die Grundannahme Kümmels, symbolisierte die gesellschaftliche Positionierung zum ehemaligen chef de l'État français sowie zum seinerzeitigen Präsidenten der Republik von Salò: Sie rückte die Kollaborateure in ein bestimmtes Verhältnis zu Staat und Nation und präjudizierte die Art, wie ihrer gedacht werden sollte. Da der Maréchal und ,Held von Verdun' ebenso wie der Duce jedoch noch immer von einem beträchtlichen Teil der Bevölkerung verehrt wurden, war die Bestattungsfrage über Jahrzehnte gesellschaftlich umkämpft. Der Diebstahl der Leichen durch neofaschistische Anhänger Mussolinis (1946) beziehungsweise rechtsgerichtete Pétainisten (1973) symbolisier-

C. C. Gatzka $(\bowtie)$

Albert-Ludwigs-Universität Freiburg, Freiburg i. Br., Deutschland

E-Mail: claudia.gatzka@geschichte.uni-freiburg.de 